\title{
Toeplitz-Superposition Operators on Analytic Bloch Spaces
}

\author{
M. A. Bakhit $\mathbb{D}^{1}$ and A. El-Sayed Ahmed $\mathbb{D}^{2}$ \\ ${ }^{1}$ Faculty of Science, Math. Dept, Jazan University, Jazan, Saudi Arabia \\ ${ }^{2}$ Mathematics Department, Faculty of Science, Taif University, P.O. Box 11099, Taif 21944, Saudi Arabia \\ Correspondence should be addressed to A. El-Sayed Ahmed; ahsayed80@hotmail.com
}

Received 1 March 2021; Revised 23 March 2021; Accepted 15 April 2021; Published 28 April 2021

Academic Editor: Gangadharan Murugusundaramoorthy

Copyright (C) 2021 M. A. Bakhit and A. El-Sayed Ahmed. This is an open access article distributed under the Creative Commons Attribution License, which permits unrestricted use, distribution, and reproduction in any medium, provided the original work is properly cited.

The important purpose of this current work is to study a new class of operators, the so-called Toeplitz-superposition operators as an expansion of the weighted known composition operators, induced by such continuous entire functions mapping on bounded specific sets. Minutely, we have deeply discussed the conditions for boundedness of this new type of operators between certain types of some holomorphic Bloch classes with some specific values of the weighted functions.

\section{Introduction}

Fundamentals of the needed analytic function spaces as well as the types of concerned operators are briefly introduced. The paper focuses first on the concerned setting of certain classes of function spaces and the new defined operator, which in turn is motivated essentially by some certain classical concepts of known operators such as superposition operators as well as Toeplitz operator. There is an emphasis in the concerned paper on intensive tying together the needed type of analytic function spaces and the concerned operators, to illustrate the roles of the obtained results.

All of the needed information to justify the target of this research is collected in this concerned section. Moreover, here, basic concerned concepts, the Bloch space of analytictype, certain needed concerned lemmas, and superposition and Toeplitz operators are presented.

Let $\mathbb{D}=\{z \in \mathbb{C}:|z|<1\}$ be the open unit disk in $\mathbb{C}$, and let $\mathscr{H}(\mathbb{D})$ denote the class of all analytic functions in $\mathbb{D}$. Let $d A$ $(z)=d x d y$ denote the concerned Lebesgue measures on $\mathbb{D}$.

Numerous intensive studies on analytic Bloch-type spaces are researched in literature (see [1-5] and others).
Let $h \in \mathscr{H}(\mathbb{D})$ and $0<b<\infty$, the $b$-Bloch space $\mathscr{B}^{b}$ is defined by

$$
\mathscr{B}^{b}=\left\{f \in \mathscr{H}(\mathbb{D}):\|h\|_{\mathscr{R}^{b}}=\sup _{\zeta \in \mathbb{D}}\left(1-|\zeta|^{2}\right)^{b}\left|f^{\prime}(\zeta)\right|<\infty\right\} .
$$

The space $\mathscr{B}^{1}$ is called the Bloch space and denoted by $\mathscr{B}$ (see [3]). in [6].

The following interesting needed lemma has been proved

Lemma 1. For a given $0<a<\infty$, let the function $h \in \mathscr{B}^{a}$. Then, we have

$$
|h(\zeta)| \lesssim \begin{cases}\|h\|_{\mathscr{B}^{a}}, & \text { if } 0<a<1 ; \\ \|h\|_{\mathscr{B}^{a}} \ln \frac{e}{1-|\zeta|^{2}}, & \text { if } a=1 ; \\ \frac{\|h\|_{\mathscr{B}^{a}}}{\left(1-|\zeta|^{2}\right)^{a-1}}, & \text { if } a>1 .\end{cases}
$$


The following useful integral estimate is well known and can be found in [7].

Lemma 2. Let $s>0$ and $t>-1$. Then

$$
\int_{\mathbb{D}} \frac{\left(1-|w|^{2}\right)^{t} d A(w)}{|1-\bar{z} w|^{2+t+s}} \lesssim \frac{1}{\left(1-|z|^{2}\right)^{s}}, \quad \text { for all } \quad z \in \mathbb{D} \text {. }
$$

For $a>-1$ and $p \in(0, \infty)$, the weighted Bergman spaces $\mathscr{A}_{a}^{P}(\mathbb{D})$ is the space of all functions $h \in \mathscr{H}(\mathbb{D})$, for which

$\|h\|_{\mathscr{A}_{a}^{p}}^{p}=\int_{\mathbb{D}}|h(\zeta)|^{p} d A_{a}(\zeta)<\infty$, where $\quad d A_{a}(\zeta)=\left(1-|\zeta|^{2}\right)^{a} d A(\zeta)$.

When $a=0$, we simply write $\mathscr{A}^{P}(\mathbb{D})$ for $\mathscr{A}_{0}^{p}(\mathbb{D})$, and when $p=2, \mathscr{A}_{a}^{2}(\mathbb{D})$ is a Hilbert space. It is well known that the Bergman kernel $K_{z}(w)$ of the Hilbert space $\mathscr{A}_{a}^{2}(\mathbb{D})$ is given by $K_{z}(w)=(1-\bar{w} z)^{-a-2}$, where $z, w \in \mathbb{D}$. The Bergman projection $P_{a}$ is the orthogonal projection from $L^{2}\left(\mathbb{D}, d A_{a}\right)$ onto Hilbert space $\mathscr{A}_{a}^{2}(\mathbb{D})$, which given as:

$$
P_{a} h(z)=\int_{\mathbb{D}} K_{z}(w) h(w) d A_{a}(w) .
$$

For $a>-1$ and $h \in \mathscr{H}(\mathbb{D})$, the Toeplitz-type operator $T_{u}^{a}$ with symbol $u \in H^{\infty}(\mathbb{D})$ is defined by

$$
T_{u}^{a} h(z)=\int_{\mathbb{D}} \frac{u(w) h(w)}{(1-\bar{w} z)^{a+2}} d A_{a}(w)
$$

This paper is organized as follows: during Section 2, we have defined the Toeplitz-superposition operators on the normed (metric) subspaces. Throughout Section 3, we establish the conditions for the Toeplitz-superposition operators to be bounded from $a$-Bloch space $\mathscr{B}^{a}$ into $b$-Bloch space $\mathscr{B}^{b}$, in the case $a \in(0,1)$ and $b>a$ or $b<a$. Section 4 is devoted to a study the boundedness of Toeplitzsuperposition operators between weighted Bloch spaces in the case $0<a \leq b$ or $a=0, b>0$.

Remark 3. It is concerned remarkable to say that two concerned quantities $N_{h}$ and $N_{h}^{*}$, where both depending on the concerned function $h \in \mathscr{H}(\mathbb{D})$, the expression $N_{h} \lesssim N_{h}^{*}$, can be satisfied when we have a concerned positive constant $C_{1}$, for which $N_{h} \leq C_{1} N_{h}^{*}$. When $N_{h}^{*} \lesssim N_{h} \lesssim N_{h}^{*}$, the expression $N_{h} \approx N_{h}^{*}$ can be written to say that there is an equivalence relation between the concerned quantities $N_{h}$ and $N_{h}^{*}$. Furthermore, when $N_{h} \approx N_{h}^{*}$, we deduce that $N_{h}<\infty \Leftrightarrow N_{h}^{*}$ $<\infty$.

\section{Toeplitz-Superposition Operators}

Let $\mathscr{E}(\mathbb{C})$ denote the set of all entire functions on the complex plane $\mathbb{C}$. For a function $\phi \in \mathscr{E}(\mathbb{C})$, the superposition operator $S_{\phi}: \mathscr{H}(\mathbb{D}) \rightarrow \mathscr{H}(\mathbb{D})$ is defined by $S_{\phi}(h)=(\phi \circ h)$. Moreover, if $u \in \mathscr{H}(\mathbb{D})$ and $\phi \in \mathscr{E}(\mathbb{C})$, the weighted superposition oper- ator $S_{\phi, u}: \mathscr{H}(\mathbb{D}) \rightarrow \mathscr{H}(\mathbb{D})$ is defined by $S_{\phi, u}(h)(z)=u(z) \phi($ $h(z))$, for all $h \in \mathscr{H}(\mathbb{D})$ and $z \in \mathbb{D}$. Note that, if $u(z)=1$, then $S_{\phi, u}=S_{\phi}$, for any $z \in \mathbb{D}$.

For any normed subspace $X \subset \mathscr{H}(\mathbb{D})$, we will consider the set $\mathscr{K}(X)$, defined by

$$
\mathscr{K}(X)=\{h \in X: \phi \circ h \in X, \quad \text { where } \quad \phi \in \mathscr{E}(\mathbb{C})\}
$$

Now, we define the Toeplitz-superposition operators acting on $\mathscr{H}(\mathbb{D})$.

Definition 4. Let two functions $\phi \in \mathscr{E}(\mathbb{C})$ and $u \in \mathscr{H}(\mathbb{D})$. Then, the Toeplitz-superposition operators $T_{u} S_{\phi}$ on the normed (metric) subspace $X$ are given by

$$
T_{u} S_{\phi}(h)=T_{u}(\phi \circ h)=P(u \cdot(\phi \circ h)), \quad \text { for all } h \in \mathscr{K}(X) .
$$

Let $\alpha, \beta$ be the scalers if $\phi$ is a fixed entire function and $u, v \in \mathscr{H}(\mathbb{D})$. Then, from the definition of Toeplitzsuperposition operators, we have

$$
\begin{aligned}
T_{\alpha u+\beta v} S_{\phi}(h) & =T_{\alpha u+\beta v}(\phi \circ h)=P(\alpha u \cdot(\phi \circ h)+\beta v \cdot(\phi \circ h)) \\
& =\alpha P(u \cdot(\phi \circ h))+\beta P(v \cdot(\phi \circ h)) \\
& =\alpha T_{u} S_{\phi}(h)+\beta T_{v} S_{\phi}(h)
\end{aligned}
$$

which holds for all $h \in \mathscr{K}(X)$, and hence, the ToeplitzSuperposition operators are linear on the normed subspace $X$.

It can be seen that whenever $u \in \mathscr{H}(\mathbb{D})$, then, the operator $T_{u} S_{\phi}$ becomes the operator $S_{\phi, u}$. So, Toeplitzsuperposition operators can be taken as an extension of weighted superposition operators. The present paper is interested in answering the following interesting questions.

(i) Can we transform one holomorphic function space into another by what kinds of entire functions?

(ii) What are the holomorphic spaces that can be transformed one into another by certain weighted classes of entire functions such as specific analytic polynomials of a certain degree and certain entire-type functions of given type and order?

(iii) When does the holomorphic function $\varphi$ induces a Toeplitz-superposition operators to form one holomorphic function space into another?

As a concerned result, the obtained results will introduce answers of the above mentioned questions by using the class of Toeplitz-superposition operators that are acting between different classes of Bloch functions.

Also, the answers for some of these concerned questions have been introduced by several authors; the following citations can be stated for interesting and intensive studies [8-20]. 
3. Boundedness in the case $a \in(0,1)$ and $b>a$ or $b<a$

Several important discussions on boundedness property of the new operator acting on the analytic Bloch spaces are presented in this concerned section. Furthermore, some essential equivalent characterizations for its boundedness are established too.

Now, we will introduce the main results of boundedness.

Theorem 5. For $a \in(0,1)$ and $b>a$. Suppose that $u \in H^{\infty}(\mathbb{D})$ and let $\phi \in \mathscr{E}(\mathbb{C})$, with $\phi \neq 0$. Then, the Toeplitzsuperposition operator $T_{u} S_{\phi}: \mathscr{B}^{a} \rightarrow \mathscr{B}^{b}$ is bounded.

Proof. First, assume that $u \in H^{\infty}(\mathbb{D})$. Let $h \in \mathscr{B}^{b}$, since

$$
\|h\|_{\mathscr{B}^{b}} \approx \sup _{z \in \mathbb{D}}\left(1-|z|^{2}\right)^{b-1}|h(z)|,
$$

we have

$$
\begin{aligned}
\left\|T_{u} S_{\phi} h\right\|_{\mathscr{B}^{b}} & \approx \sup _{z \in \mathbb{D}}\left(1-|z|^{2}\right)^{b-1}\left|T_{u} S_{\phi} h\right| \\
& =\sup _{z \in \mathbb{D}}\left(1-|z|^{2}\right)^{b-1}\left|\int_{\mathbb{D}} \frac{u(w)(\phi \circ h)(w)}{(1-\bar{w} z)^{2}} d A(w)\right| \\
& \leq \sup _{z \in \mathbb{D}}\left(1-|z|^{2}\right)^{b-1} \int_{\mathbb{D}} \frac{|u(w)||\phi(h(w))|}{|1-\bar{w} z|^{2}} d A(w) \\
& \leq\|u\|_{H^{\infty}} \sup _{z \in \mathbb{D}}\left(1-|z|^{2}\right)^{b-1} \int_{\mathbb{D}} \frac{\mid \phi(h(w) \mid}{|1-\bar{w} z|^{2}} d A(w) .
\end{aligned}
$$

Now, let the constant $R>0$ where $h \in \mathscr{B}^{a}$ such that $\| h$ $\|_{\mathscr{B}^{a}} \leq R$, by Lemma 1 , we have $|h(z)| \leq R$. Set $R_{1}=\max _{|z|=R} \mid \phi$ $(z) \mid$, then $|\phi(h(z))| \leq R_{1}$. Since $b>a$, we have the fact that $\mathscr{B}^{a} \subset \mathscr{B}^{b}$, and since $a \in(0,1)$, we have that $\mathscr{B}^{a} \subset H^{\infty}(\mathbb{D})$. Thus,

$$
\begin{aligned}
\left\|T_{u} S_{\phi} h\right\|_{\mathscr{B}^{b}} & \lesssim R_{1}\|u\|_{H^{\infty}} \sup _{z \in \mathbb{D}}\left(1-|z|^{2}\right)^{b-1} \int_{\mathbb{D}} \frac{d A(w)}{|1-\bar{w} z|^{2}} \\
& \leqslant\|u\|_{H^{\infty}}<\|u\|_{\mathscr{B}^{a}}<\infty,
\end{aligned}
$$

where $R, R_{1}$ depended only on $a, b$, and $\phi$. This shows that $T_{u} S_{\phi}: \mathscr{B}^{a} \rightarrow \mathscr{B}^{b}$ is bounded.

Theorem 6. For $0<b<a<1$, let $u \in L^{1}(\mathbb{D})$ be harmonic and let $\phi \in \mathscr{E}(\mathbb{C})$. Then, the Toeplitz-superposition operator $T_{u}$ $S_{\phi}: \mathscr{B}^{a} \rightarrow \mathscr{B}^{b}$ is bounded if and only if $u \in H^{\infty}(\mathbb{D})$ and $\phi$ is a constant entire function.

Proof. It is trivial that if $u \in H^{\infty}(\mathbb{D})$ and $\phi$ is constant, then $T_{u} S_{\phi}: \mathscr{B}^{a} \rightarrow \mathscr{B}^{b}$ is bounded. If $\phi$ is constant, not identically 0 , and $T_{u} S_{\phi}$ maps $\mathscr{B}^{a}$ into $\mathscr{B}^{b}$ then it is clear that $u \in H^{\infty}(\mathbb{D})$. Assume now that $u \neq 0$ and $\phi$ is not constant, and set $T_{u} S_{\phi}$ maps $\mathscr{B}^{a}$ into $\mathscr{B}^{b}$. Let $h$ be the constant function defined by $h(\zeta)=\lambda$, for all $\zeta \in \mathbb{D}$, such that $\phi(\lambda) \neq 0$. Since $h \in \mathscr{B}^{a}$, it follows that $T_{u} S_{\phi} h(\zeta)=T_{u} \phi(\lambda) \in \mathscr{B}^{b}$. This implies that $u$ $\in \mathscr{B}^{b} \subset H^{\infty}(\mathbb{D})$, since $0<b<1$. Finally, since $\phi$ is not constant, then there is a disk $\left|w-w_{0}\right|<\varepsilon$ and $\delta>0$, on which $|\phi(w)|>\delta|w|$. Set the test function $h_{0}(w)=w_{0}+r(1-w)^{1-a}$ $\in \mathscr{B}^{a}$. Then, for all $w \in \mathbb{D}$, we have

$$
\begin{aligned}
\left\|T_{u} S_{\phi} h_{0}\right\|_{\mathscr{B}^{b}} & \approx \sup _{z \in \mathbb{D}}\left(1-|z|^{2}\right)^{b-1}\left|T_{u} S_{\phi} h_{0}\right| \\
& =\sup _{z \in \mathbb{D}}\left(1-|z|^{2}\right)^{b-1}\left|\int_{\mathbb{D}} \frac{u(w)\left(\phi \circ h_{0}\right)(w)}{(1-\bar{w} z)^{2}} d A(w)\right| \\
& \geq \sup _{z \in \mathbb{D}}\left(1-|z|^{2}\right)^{b-1} \int_{\mathbb{D}} \frac{|u(w)|}{|1-w|^{a}|1-\bar{w} z|^{2}} d A(w) .
\end{aligned}
$$

But, along with the positive radius, we get $|u(w)| /($ $\left.|1-w|^{a}|1-\bar{w} z|^{2}\right) \rightarrow \infty$, as $w \rightarrow 1$. This shows that $T_{u} S_{\phi}$ $: \mathscr{B}^{a} \rightarrow \mathscr{B}^{b}$ is not bounded.

4. Boundedness in the case $0<a \leq b$ or $a=0, b>0$

Theorem 7. For $a>0$, let $u \in L^{1}(\mathbb{D})$ be harmonic and let $\phi$ $\in \mathscr{E}(\mathbb{C})$. Then, $T_{u} S_{\phi}$ is bounded on $\mathscr{B}^{a}$ if and only if $u \in$ $H^{\infty}(\mathbb{D})$ and $\phi$ is an affine function (linear function plus a translation).

Proof. First, suppose that $u \in H^{\infty}(\mathbb{D})$ and $\phi$ is an affine function. It is easy to explain $T_{u} S_{\phi}$ is bounded from $\mathscr{B}^{a}$ into itself.

On the other hand, assume that $u \in H^{\infty}(\mathbb{D})$ and $\phi \in \mathscr{E}(\mathbb{C})$ does not linear function. Then, by using the Cauchy estimates for $\phi \in \mathscr{E}(\mathbb{C})$, we can find a sequence $\left\{w_{n}\right\} \subset \mathbb{C}$, for each $n \in \mathbb{N}$ such that $\left|w_{n}\right| \rightarrow \infty$ as $n \rightarrow \infty$ and $\left|\phi\left(w_{n}\right)\right|=$ $\max _{|w|=n}|\phi(w)| \gtrsim\left|w_{n}\right|^{2}$. Also, since the weight $\left(1-|\zeta|^{2}\right)^{a}$ is typical, we can find a sequence of points $\left\{z_{n}\right\} \subset \mathbb{D}$ such that $\left|z_{n}\right| \rightarrow 1^{-}$, with $\left(0.5<\left|z_{n}\right|<1\right)$ and such that $\left(1-\left|z_{n}\right|\right) \mid$ $w_{n} \mid=1$, for all $n \in \mathbb{N}$. Now consider the sequence of functions $\left\{h_{n}\right\}$ contained in $\mathscr{B}^{a}$ satisfies $\left\|h_{n}\right\|_{\mathscr{B}^{a}} \leq 1$ and $\left|h_{n}\left(z_{n}\right)\right|=\left|w_{n}\right|$. Furthermore, we can suppose that $h_{n}$ $\left(z_{n}\right)=w_{n}$. Hence,

$$
\begin{aligned}
\left\|T_{u} S_{\phi}\left(h_{n}\right)\right\|_{\mathscr{B}^{b}} & \approx \sup _{z_{n} \in \mathbb{D}}\left(1-\left|z_{n}\right|^{2}\right)^{b-1}\left|T_{u} \phi\left(h_{n}\left(z_{n}\right)\right)\right| \\
& \geq\|u\|_{H^{\infty}} \sup _{z \in \mathbb{D}}\left(1-\left|z_{n}\right|^{2}\right)^{b-1} \int_{\mathbb{D}} \frac{\left|w_{n}\right|^{2}}{\left|1-\bar{w} z_{n}\right|^{2}} d A(w) \\
& \geq\|u\|_{H^{\infty}} \sup _{z \in \mathbb{D}}\left(1-\left|z_{n}\right|^{2}\right)^{b-1} \int_{\mathbb{D}} \frac{\left|w_{n}\right|}{|1-\bar{w} z|^{2}} d A(w) \\
& \rightarrow \infty, \text { as } n \rightarrow \infty .
\end{aligned}
$$

Because $\left|w_{n}\right| \rightarrow \infty$ as $n \rightarrow \infty$. This shows that $T_{u} S_{\phi}$ $: \mathscr{B}^{a} \rightarrow \mathscr{B}^{a}$ cannot be bounded if $\phi \in \mathscr{E}(\mathbb{C})$ is not a linear function. 
Theorem 8. For $0<a \leq b$, let $u \in L^{1}(\mathbb{D})$ be harmonic and let $\phi \in \mathscr{E}(\mathbb{C})$ be an increasing and continuous function. Then, $T_{u} S_{\phi}: \mathscr{B}^{a} \rightarrow \mathscr{B}^{b}$ is bounded if and only if $u \in H^{\infty}(\mathbb{D})$, and for each $\lambda \in(0,1)$, there is a positive constant $\eta$ whenever $|w|>\eta$, such that

$$
|\phi(w)| \lesssim \phi(\lambda|w|) .
$$

Proof. First, suppose that $u \in H^{\infty}(\mathbb{D})$ and (15) is true. Now, consider $R_{1}>0$ and let $h \in \mathscr{B}^{a}$ satisfy $\|h\|_{\mathscr{B}^{a}} \leq R_{1}$ and select $\lambda \in(0,1)$ such that $\lambda R_{1}<1$. Then, there is $\eta>0$, such that $|\phi(w)| \lesssim \phi(\lambda|w|)$, whenever $|w|>\eta$. Thus, since $\bar{D}_{R}=\{w$ $\in \mathbb{C}:|w| \leq R\}$ is a compact set and $\phi \in \mathscr{E}(\mathbb{C})$ is a continuous function, we can assume that $|\phi(w)| \leq 1$, for all $w \in \bar{D}_{R}$. Hence,

$$
\begin{aligned}
& \left\|T_{u} S_{\phi} h\right\|_{\mathscr{B}^{b}} \approx \sup _{z_{n} \in \mathbb{D}}\left(1-\left|z_{n}\right|^{2}\right)^{b-1}\left|T_{u} \phi(h(z))\right| \\
& =\sup _{\{z \in \mathbb{D},|h(w)| \leq R\}}\left(1-|z|^{2}\right)^{b-1}\left|T_{u} S_{\phi} h\right| \\
& +\sup _{\{z \in \mathbb{D},|h(w)|>R\}}\left(1-|z|^{2}\right)^{b-1}\left|T_{u} \phi(h(z))\right| \\
& \leqslant\|u\|_{H^{\infty}} \sup _{z \in \mathbb{D}}\left(1-|z|^{2}\right)^{b-1} \int_{\mathbb{D}} \frac{d A(w)}{|1-\bar{w} z|^{2}} \\
& +\|u\|_{H^{\infty}} \sup _{\{z \in \mathbb{D},|h(w)|>R\}}\left(1-|z|^{2}\right)^{b-1} \int_{\mathbb{D}} \phi \\
& \cdot\left(\lambda\left|\frac{\|h\|_{\mathscr{B}^{a}}}{\left(1-|w|^{2}\right)^{a}}\right|\right) \frac{d A(w)}{|1-\bar{w} z|^{2}} \\
& \lesssim\|u\|_{H^{\infty}}+\|u\|_{H^{\infty}} \sup _{z \in \mathbb{D}}\left(1-|z|^{2}\right)^{b-1} \int_{\mathbb{D}} \phi \\
& \cdot\left(\lambda\left|\frac{1}{\left(1-|w|^{2}\right)^{a}}\right|\right) \frac{d A(w)}{|1-\bar{w} z|^{2}} \text {. }
\end{aligned}
$$

Using that the function $\phi$ is increasing and the fact that $\lambda<1$, we have

$$
\begin{aligned}
\left\|T_{u} S_{\phi} h\right\|_{\mathscr{B}^{b}} & \leqslant\|u\|_{H^{\infty}}+\|u\|_{H^{\infty}} \sup _{z \in \mathbb{D}}\left(1-|z|^{2}\right)^{b-1} \int_{\mathbb{D}} \frac{d A(w)}{|1-\bar{w} z|^{2}} \\
& \leqslant\|u\|_{H^{\infty}} .
\end{aligned}
$$

This shows that $T_{u} S_{\phi}: \mathscr{B}^{a} \rightarrow \mathscr{B}^{b}$ is bounded.

On the other hand, assume that $u \in H^{\infty}(\mathbb{D})$ and $\phi \in \mathscr{E}($ $\mathbb{C})$ does not satisfy (15). Then, we can find $\lambda_{1} \in(0,1)$ and a sequence $\left\{w_{n}\right\} \subset \mathbb{C}$ such that $\left|w_{n}\right| \rightarrow \infty$ as $n \rightarrow \infty$ and $\mid \phi($ $\left.w_{n}\right) \mid \geq \phi\left(\lambda_{1}\left|w_{n}\right|\right)$, for all $n \in \mathbb{N}$. Since the weight $\left(1-|z|^{2}\right)^{a}$ is typical, we can find a sequence of points $\left\{z_{n}\right\}$ $\subset \mathbb{D}$ such that $\left|z_{n}\right| \rightarrow 1^{-}$as $n \rightarrow \infty$. Thus, we can consider a sequence of functions $\left\{h_{n}\right\}$ contained in $\mathscr{B}^{a}$ satisfies $\| h_{n}$ $\|_{\mathscr{B}^{a}} \leq 1$ and $\left|h_{n}\left(z_{n}\right)\right| \leq\left|w_{n}\right|$. Now, let $z \in \mathbb{D}$ and set the function $f_{n}(z)=w_{n} h_{n}(z) / h_{n}\left(z_{n}\right)$ for all $n \in \mathbb{N}$. Then, we have $f_{n}$ $\left(z_{n}\right)=w_{n}$ and $\left\|f_{n}\right\|_{\mathscr{B}^{a}} \lesssim 1$. For large enough $n \in \mathbb{N}$, we obtain

$$
\begin{aligned}
\left\|T_{u} S_{\phi} f_{n}\right\|_{\mathscr{B}^{b}} \approx \sup _{z_{n} \in \mathbb{D}}\left(1-\left|z_{n}\right|^{2}\right)^{b-1}\left|T_{u} S_{\phi} f_{n}\left(z_{n}\right)\right| \\
\approx \sup _{z_{n} \in \mathbb{D}}\left(1-\left|z_{n}\right|^{2}\right)^{b-1} \int_{\mathbb{D}} \frac{|u(w)|\left|\phi\left(w_{n}\right)\right|}{\left|1-\bar{w} z_{n}\right|^{2}} d A(w) \\
\approx\|u\|_{H^{\infty}} \sup _{z_{n} \in \mathbb{D}}\left(1-\left|z_{n}\right|^{2}\right)^{b-1} \int_{\mathbb{D}} \frac{\phi\left(\lambda_{1}\left|w_{n}\right|\right)}{\left|1-\bar{w} z_{n}\right|^{2}} d A(w) \\
\gtrsim\|u\|_{H^{\infty}} \sup _{z_{n} \in \mathbb{D}}\left(1-\left|z_{n}\right|^{2}\right)^{b-1} \int_{\mathbb{D}} \phi\left(\left|\frac{1}{\left(1-\left|w_{n}\right|^{2}\right)^{a}}\right|\right) \\
\quad \cdot \frac{d A(w)}{\left|1-\bar{w} z_{n}\right|^{2}} \rightarrow \infty, a s n \rightarrow \infty .
\end{aligned}
$$

Then, we conclude that $T_{u} S_{\phi}: \mathscr{B}^{a} \rightarrow \mathscr{B}^{b}$ cannot be bounded.

Theorem 9. For $1<a \leq b$, let $u \in L^{1}(\mathbb{D})$ be harmonic and let $\phi \in \mathscr{E}(\mathbb{C})$. Then, the following are equivalent:

(i) $T_{u} S_{\phi}$ maps $\mathscr{B}^{a}$ into $\mathscr{B}^{b}$

(ii) $u \in H^{\infty}(\mathbb{D})$ and $\phi$ is a polynomial of degree at most $b-1 / a-1$

(iii) $T_{u} S_{\phi}: \mathscr{B}^{a} \rightarrow \mathscr{B}^{b}$ is bounded

Proof. First, suppose that (i) holds, let $\phi=z^{n}$ be a polynomial with $n \leq(b-1) /(a-1)$, then, we have that $u \in H^{\infty}(\mathbb{D})$.

Now, suppose that the entire function $\phi$ is a polynomial of degree $m>b-1 / a-1$. Then, for an integer $n$ and a positive constant $\delta$, there is a sequence $z_{n} \rightarrow \infty$ such that $\mid \phi\left(z_{n}\right)$ $\left.|\geq \delta| z_{n}\right|^{m}$. We may assume without losing generality that $\left|z_{n}\right|>1$ and $\left|\arg z_{n}\right|<\min \{a \pi / 4, \pi / 2\}$, for an integer $n$. Now, we let $h_{a}(w)=(1-w)^{1-a} \in \mathscr{B}^{a}$, then, we show that $T_{u} S_{\phi} h_{a} \notin \mathscr{B}^{b}$. The point $w_{n}=1-\left(z_{n}\right)^{-1 / a}$ such that $\mid 1-w_{n}$ $\mid<1$ and $\left|\arg \left(1-w_{n}\right)\right|<\pi / 4$, and satisfies that $\left|1-w_{n}\right| \lesssim(1$ $\left.-w_{n}\right)$, for an integer $n$. Thus, we have

$$
\begin{aligned}
(1 & \left.-\left|z_{n}\right|^{2}\right)^{b-1}\left|T_{u} S_{\phi} h_{a}\left(z_{n}\right)\right| \\
& =\left(1-\left|z_{n}\right|^{2}\right)^{b-1}\left|\int_{\mathbb{D}} \frac{u(w) \phi\left(h_{a}(w)\right)}{\left(1-\bar{w} z_{n}\right)^{2}} d A(w)\right| \\
& \geq\left(1-\left|z_{n}\right|^{2}\right)^{b-1} \int_{\mathbb{D}} \frac{\delta|u(w)|}{\left|1-z_{n}\right|^{m a}\left|1-\bar{w} z_{n}\right|^{2}} d A(w) \\
& \geq \int_{\mathbb{D}} \frac{\delta|u(w)|}{\left|1-z_{n}\right|^{m a-m-b+1}|1-w|^{a}\left|1-\bar{w} z_{n}\right|^{2}} d A(w) .
\end{aligned}
$$

Since $m a-m-b+1>0$, then, we obtain

$$
\left(1-\left|z_{n}\right|^{2}\right)^{b-1}\left|T_{u} S_{\phi} h_{a}\left(z_{n}\right)\right| \rightarrow \infty, \text { as } n \rightarrow \infty .
$$

This implies that $T_{u} S_{\phi} h_{a} \notin \mathscr{B}^{b}$. Based on the above it is clear that (i) $\Rightarrow$ (ii). 
Second, assume that $u \in H^{\infty}(\mathbb{D})$ and $\phi=z^{n}$ are a polynomial of degree $n \leq(b-1) /(a-1)$. For all $h \in \mathscr{B}^{a}$ and $b-1$ $-n a-n \geq 0$, by Lemma 1 and 2, where $n$ is bounded, we have

$$
\begin{aligned}
\left\|T_{u} S_{\phi} h\right\|_{\mathscr{B}^{b}} & \approx \sup _{z \in \mathbb{D}}\left(1-|z|^{2}\right)^{b-1}\left|T_{u} S_{\phi} h(z)\right| \\
& =\sup _{z \in \mathbb{D}}\left(1-|z|^{2}\right)^{b-1}\left|\int_{\mathbb{D}} \frac{u(w)(\phi \circ h)(w)}{(1-\bar{w} z)^{2}} d A(w)\right| \\
& \leq\|u\|_{H^{\infty}} \sup _{z \in \mathbb{D}}\left(1-|z|^{2}\right)^{b-1} \int_{\mathbb{D}} \frac{|h(w)|^{n}}{|1-\bar{w} z|^{2}} d A(w) \\
& \leq\|u\|_{H^{\infty}}\|h\|_{\mathscr{B}^{a}}^{n} \sup _{z \in \mathbb{D}}\left(1-|z|^{2}\right)^{b-1-n a-n} \int_{\mathbb{D}} \frac{d A(w)}{|1-\bar{w} z|^{2}} \\
& \leq\|u\|_{H^{\infty}}\|h\|_{\mathscr{B}^{a}}^{n} .
\end{aligned}
$$

This shows that (ii) $\Rightarrow$ (iii). Thus, the proof has been completed.

Theorem 10. For $b>-1$, let $u \in L^{1}(\mathbb{D})$ be harmonic and let $\phi \in \mathscr{E}(\mathbb{C})$, with order $\rho$ and type $\tau$. Then, the following are equivalent:

(i) $T_{u} S_{\phi}$ maps $\mathscr{B}$ into $\mathscr{B}^{b}$

(ii) $u \in H^{\infty}(\mathbb{D})$ and $\phi \in \mathscr{E}(\mathbb{C})$ with $\rho<1$ or (with $\rho=1$ and $\tau=0)$;

(iii) $T_{u} S_{\phi}: \mathscr{B} \rightarrow \mathscr{B}^{b}$ is bounded

Proof. First, assume that $T_{u} S_{\phi}$ maps $\mathscr{B}$ into $\mathscr{B}^{b}$. Now, we assume on the antithesis that (ii) does not hold. Then, the function $\phi \in \mathscr{E}(\mathbb{C})$ with $\rho>1$ or (with $\rho=1$ and $\tau>0$ ). Thus, there is a positive constant $\lambda$ and a sequence $\left\{w_{n}\right\}$ of complex numbers such that $\left|w_{n}\right| \rightarrow \infty$ and

$$
\left|\phi\left(w_{n}\right)\right| \geq \exp \left(\lambda\left|w_{n}\right|\right), \quad \text { for } \quad \text { any } \quad n \in \mathbb{N} \text {. }
$$

Hence, as in the proof of Theorem 8 , we can consider the sequence $\left\{w_{n}\right\} \subset \mathbb{D}$ and $\left\{h_{n}\right\} \subset \mathscr{B}$ satisfies $\left\|h_{n}\right\|_{\mathscr{B}} \leq 1$ and $\left|h_{n}\left(z_{n}\right)\right| \leq\left|w_{n}\right|$. Now, let $z \in \mathbb{D}$ and set the function $f_{n}(z)$ $=w_{n} h_{n}(z) / h_{n}\left(z_{n}\right)$ for all $n \in \mathbb{N}$. Then, we have $f_{n}\left(z_{n}\right)=w_{n}$ and $\left\|f_{n}\right\|_{\mathscr{B}} \lesssim 1$. For large enough, $n \in \mathbb{N}$, since $\left|w_{n}\right| \rightarrow \infty$, we obtain

$$
\begin{aligned}
\left\|T_{u} S_{\phi} f_{n}\right\|_{\mathscr{B}^{b}} & \approx \sup _{z \in \mathbb{D}}\left(1-\left|z_{n}\right|^{2}\right)^{b-1}\left|T_{u} S_{\phi} f_{n}\left(z_{n}\right)\right| \\
& \geq \sup _{z \in \mathbb{D}}\left(1-\left|z_{n}\right|^{2}\right)^{b-1} \int_{\mathbb{D}} \frac{|u(w)|\left|\phi\left(w_{n}\right)\right|}{\left|1-\bar{w} z_{n}\right|^{2}} d A(w) \\
& \geq\|u\|_{H^{\infty}} \sup _{z \in \mathbb{D}}\left(1-\left|z_{n}\right|^{2}\right)^{b-1} \int_{\mathbb{D}} \frac{\exp \left(\lambda_{1}\left|w_{n}\right|\right)}{\left|1-\bar{w} z_{n}\right|^{2}} d A(w) \\
& \rightarrow \infty, a s n \rightarrow \infty .
\end{aligned}
$$

Then, we conclude that $T_{u} S_{\phi}: \mathscr{B} \rightarrow \mathscr{B}^{b}$ cannot be bounded. Based on the above results, it is clear that (i) $\Rightarrow$ (ii).

Second, set $M(t, \phi)=\max _{|w|=t}|\phi(w)|$, where $t \geq 0$, the order $\rho$ of $\phi \in \mathscr{E}(\mathbb{C})$ is

$$
\rho=\limsup _{t \rightarrow \infty} \frac{\log \log M(t, \phi)}{\log t} .
$$

If $0<\rho<\infty$, then the type $\tau$ of $\phi \in \mathscr{E}(\mathbb{C})$ is

$$
\tau=\limsup _{t \rightarrow \infty} \frac{\log M(t, \phi)}{t^{\rho}} .
$$

For given $\lambda=b / R>0$, the condition (ii) implies that (see for example [18])

$$
|\phi(w)| \lesssim \exp (\lambda|w|), \quad \text { for any } \quad w \in \mathbb{C} .
$$

Moreover, for a function $h \in \mathscr{B}$, with $\|h\|_{\mathscr{B}} \lesssim 1$, we know that

$$
|h(w)| \lesssim\left(1+\log \frac{1}{1-|w|}\right), \quad \text { for } \quad w \in \mathbb{D} .
$$

Then,

$$
|\phi(h(w))| \leq \exp (\lambda|h(w)|) \leq\left(\frac{e}{1-|w|}\right)^{b} \leq(2 e)^{b} .
$$

Thus, we have

$$
\begin{aligned}
\left\|T_{u} S_{\phi} h\right\|_{\mathscr{B}^{b}} & \approx \sup _{z \in \mathbb{D}}\left(1-|z|^{2}\right)^{b-1}\left|T_{u} S_{\phi} h(z)\right| \\
& \leq(2 e)^{b}\|u\|_{H^{\infty}} \sup _{z \in \mathbb{D}}\left(1-|z|^{2}\right)^{b-1} \int_{\mathbb{D}} \frac{d A(w)}{|1-\bar{w} z|^{2}} \\
& \leq(2 e)^{b}\|u\|_{H^{\infty}}<\infty .
\end{aligned}
$$

This shows that $T_{u} S_{\phi}: \mathscr{B} \rightarrow \mathscr{B}^{b}$ is bounded. So, (ii) $\Rightarrow$ (iii).

\section{Conclusion and Future Study}

This manuscript deals with a radical study of a concerned class of Toeplitz superposition operators acting between some certain classes of analytic function spaces of Blochtype. Global discussions of the boundedness property of the new class of operators are presented class of the univalent Bloch functions. All concerned entire functions which transform a class of holomorphic Bloch-type spaces into another using the so-called Toeplitz superposition operators in terms of their order and type or the degree of polynomials are characterized in this paper. Moreover, all the defined Toeplitz-superposition operators induced by concerned entire functions are cleared to be bounded actually. We have cleared that for two spaces of normed-type which belonging 
to $\mathscr{H}(\mathbb{D})$, where $X=\mathscr{B}^{a}$ and $Y=\mathscr{B}^{b}$, we can find certain concerned functions $\phi$ and $u$, with $\phi \in \mathscr{E}(\mathbb{C})$ and $u \in \mathscr{H}(\mathbb{D})$, for which the newly Toeplitz-superposition operators $T_{u} S_{\phi}$ can map $\mathscr{B}^{a}$ into $\mathscr{B}^{b}$ for some specific values of $a$ and $b$. Furthermore, the operator $T_{u} S_{\phi}: X \rightarrow Y$ is shown to be actually bounded.

\section{Data Availability}

The data is not applicable to this concerned article as no concerned data sets were created or used through this concerned study.

\section{Conflicts of Interest}

The authors declare that they have no competing interest.

\section{Acknowledgments}

The authors would like to thank Taif University Researchers supporting Project number (TURSP-2020/159), Taif University-Saudi Arabia. The authors would like to thank the anonymous concerned reviewers for their valuable remarks on this study.

\section{References}

[1] I. Abbasi and H. Vaezi, "Estimates of essential norm of generalized weighted composition operators from Bloch type spaces to nth weighted type spaces," Mathematica Slovaca, vol. 70, no. 1, pp. 71-80, 2020.

[2] I. Abbasi, S. Li, and H. Vaezi, "Weighted composition operators from the Bloch space tonth weighted-typespaces," Turkish Journal of Mathematics, vol. 44, no. 1, pp. 108-117, 2020.

[3] J. Arazy, D. Fisher, and J. Peetre, "Möbius invariant function spaces," Journal fur die Reine und Angewandte Mathematik, vol. 363, pp. 110-145, 1986.

[4] S. Kumar and S. K. Sahoo, "Properties of b-Cesáro operators on $\alpha$-Bloch space," Rocky Mountain Journal of Mathematics, vol. 50, no. 5, pp. 1723-1746, 2020.

[5] M. Pavlović, "Function classes on the unit disc: an introduction," in 1441.30002 De Gruyter Studies in Mathematics 52, De Gruyter, Berlin, 2nd revised and extended edition, 2019.

[6] S. Stević, "On an integral operator on the unit ball in $\mathbb{C}^{n}$," Journal of Inequalities and Applications, vol. 1, 88 pages, 2005.

[7] K. Zhu, "Bloch type spaces of analytic functions," The Rocky Mountain Journal of Mathematics, vol. 23, pp. 1143-1177, 1993.

[8] A. El-Sayed Ahmed and S. Omran, "Weighted superposition operators in some analytic function spaces," Journal of Computational Analysis and Applications, vol. 15, no. 6, pp. 9961005, 2013.

[9] V. Álvarez, M. A. Márquez, and D. Vukotić, "Superposition operators between the Bloch space and Bergman spaces," Arkiv för Matematik, vol. 42, no. 2, pp. 205-216, 2004.

[10] J. Appell and P. P. Zabrejko, Nonlinear Superposition Operators, Cambridge University Press, Cambridge, 2012.

[11] S. M. Buckley, J. L. Fernández, and D. Vukotić, "Superposition operators on Dirichlet type spaces," Papers on Analysis: A volume dedicated to Olli Martio on the Occasion of his 60th Birth- day, Report University of Jyväskylä Department of Mathematics and Statistics, vol. 83, University of Jyväskylä, Jyväskylä, 2001.

[12] S. M. Buckley and D. Vukotić, "Univalent interpolation in Besov spaces and superposition into Bergman spaces," Potential Analysis, vol. 29, no. 1, pp. 1-16, 2008.

[13] G. A. Cámera, "Nonlinear superposition on spaces of analytic functions," in Harmonic Analysis and Operator Theory (Caracas, 1994), Contemporary Mathematics, vol. 189, pp. 103-116, American Mathematical Society, Providence, RI, 1995.

[14] G. A. Cámera and J. Gimenez, "The nonlinear superposition operator acting on Bergman spaces," Compositio Mathematica, vol. 93, pp. 23-35, 1994.

[15] Z. J. Jiang, T. Wang, J. Liu, J. Luo, and T. Song, "Weighted superposition operators from Zygmund space to $\mu$-Bloch spaces," Journal of Computational Analysis and Applications, vol. 23, no. 3, pp. 487-495, 2017.

[16] Y. X. Liang and Z. H. Zhou, "The nonlinear superposition operators between Zygmund-type and Bloch-type spaces," Mediterranean Journal of Mathematics, vol. 16, no. 2, 2019.

[17] R. J. Malavé and J. C. R. Fernández, "Superposition operators between logarithmic Bloch spaces," Rendiconti del Circolo Matematico di Palermo Series, vol. 68, no. 1, pp. 105-121, 2019.

[18] R. J. Malavé and J. C. R. Fernández, “The integral weight and superposition operators between Bloch-type spaces," Bulletin of the Malaysian Mathematical Sciences Society, vol. 43, no. 4, pp. 3035-3047, 2020.

[19] C. Xiong, "Superposition operators between $Q_{p}$ spaces and Bloch-type spaces," Complex Variables, Theory and Application: An International Journal, vol. 50, no. 12, pp. 935-938, 2005.

[20] W. Xu, "Superposition operators on Bloch-type spaces," Computational Methods and Function Theory, vol. 7, no. 2, pp. 501-507, 2007. 